\title{
CHECKLIST OF EARTHWORMS OF WESTERN HIMALAYA, INDIA
}

\author{
R. Paliwal ${ }^{1}$ and J.M. Julka ${ }^{2}$ \\ Zoological Survey of India, High Altitude Zoology Field Station, Solan, Himachal Pradesh 173211, India \\ Email: ${ }^{1}$ rahul_solan1956@yahoo.com; ${ }^{2}$ jmjulka@hotmail.com (Corresponding author)
}

\begin{abstract}
Aвstract
A total of 51 species of earthworms belonging to 23 genera and seven families from the western Himalaya are listed in this checklist. Detailed distributional records at district level under the western Himalayan states of Jammu \& Kashmir, Himachal Pradesh and Uttaranchal are provided.
\end{abstract}

\section{KEYWORDS}

Earthworms, Western Himalaya, checklist, distribution.

Earthworms have drawn the attention of several taxonomists all over the world because of their immense importance in soil improvement and enhancing soil fertility. Bourne (1889) was the first to explore earthworm fauna of the western Himalaya. Subsequently, the knowledge on earthworm diversity of this region has been enriched by Fedarb (1898), Michaelsen (1907, 1909) and Stephenson (1914, 1916, 1922). Stephenson (1923) listed 28 species from the western Himalaya in his famous Fauna of British India volume on Oligochaeta. Cernosvitov (1937), Gates (1945, 1951), Soota (1970), Sharma and Kaul (1974), Julka (1979, 1981, 1988, 1995, 1999), Soota and Halder (1980), Julka and Paliwal (1993, 1994, 1995, 2000), and Halder and Ghosh (1997) have further supplemented the knowledge on the western Himalayan earthworm diversity. This checklist is based on previous records as well as studies on fresh material from the western Himalayan states of Jammu \& Kashmir, Himachal Pradesh and Uttaranchal. As a result, 51 species are listed from the area.

Species marked with an asterisk $(*)$ are first records from western Himalaya. Names of new locality record are italicized in the text.

\section{Family: Moniligastridae}

1. Drawida japonica (Michaelsen, 1892)

Distribution: J \& K: Doda dist.- Kishtwar, Bhaderwah, Doda; Jammu dist.- Jammu; Kathua dist.- Kathua; Poonch dist.Poonch; Rajouri dist.- Rajouri; Srinagar dist.- Srinagar; Udhampur dist.- Udhampur. H.P.: Chamba dist.- Banikhet, Khajjiar, Kalatop, Bharmour, Chaned; Hamirpur dist.Sindhari; Kangra dist.- McLeodganj; Kullu dist.- Larji, Sainj Valley (Sainj, Ropa, Lapah); Mandi dist.- Barot; Shimla dist.Ghanahatti, Shimla; Sirmour dist.- Naina Tikkar; Solan dist.Dharampur, Gambhar Bridge, Arki, Solan, Subathu, Sadhupul, Deothal, Shilli Forest, Barog. Uttaranchal: Dehra Dun dist.Chakrata, Mussoorie.

\section{Drawida nepalensis Michaelsen, 1907}

Distribution: J \& K: Budgam dist.- Ratsun. H.P.: Kangra dist.Sadwan, Dadh, Nagrota, Neugal Khud; Mandi dist.Barchhwar, Pandoh Dam, Joginder Nagar, Mohan Ghatti,
Gumma, Dak Bagda, Ner Chowk, Sunder Nagar, Hara Bagh. Uttaranchal: Almora dist.- Soni, Kausani-Almora Road, Kausani-Garur Road; Dehra Dun dist.- Dehra Dun; Naini Tal dist.- Naini Tal.

\section{Family: Lumbricidae}

3. Allolobophora eiseni (Levinsen, 1884)

Distribution: Uttaranchal: Almora dist. - Dhakuri, Dwali; Chamoli dist.- Gairoli Patali, Bedni; Naini Tal dist.- Naini Tal, Painsur.

\section{Allolobophora parva Eisen, 1874}

Distribution: J \& K: Budgam dist.- Chadura; Jammu dist.-Kokar Nag; Srinagar dist.- Gorai, Srinagar; Udhampur dist.- Mansar Lake, Balli Nalla Udhampur. H.P.: Bilaspur dist.- Kanaula, Barmala; Chamba dist.- Rakh, Khajjiar, Bhanjraroo, Gajnui, Jukiyani, Chamba; Hamirpur dist.- Heranagar, Nauli Khud, Galod, Bharnani, Man Khud Badsar; Kangra dist.- Neugal Khud, Churan Khud, Loond Khud, Multhan; Kinnaur dist.Wangtu, Pooh, Chango, Sungra, Sangla, Khursaran Nalla, Rogi; Kullu dist.- Kullu, Vashistha Kund, Tirthan Valley (Shai Ropa), Sainj Valley (Sainj, Shakti), Jiwanal, Rohtang-Marhi, Kothi, Rahla Fall; Lahaul \& Spiti dist.- Thirot, Madgram, Keylong, Shansha; Mandi dist.- Ranuki Khud, Barot, Kao; Shimla dist.- Janog Ghat, Jatog, Rajpur, Kinu, Ghanahatti, Kufri, Shimla; Sirmour dist.- Giripul, Paonta Sahib, Kheri, Haloni, Tikattu, Bandli, Rajgarh, Daluwala, Renuka Sanctuary, Saniyo Didag, Nohra; Solan dist.- Ashwini Khud, Ramshahar, Swargdwari, Nalagarh, Oachghat, Solan, Arki, Kasauli, Barog, Chhawasa, Jarash, Shilli Forest, Chail; Una dist.- Nanavi, Samoor Kalan, Kuwadi, Tutroo, Kokra. Uttaranchal: Champawat dist.- Monpokhari Forest; Dehra Dun dist.- Sahiya, Sahastradhara; Naini Tal dist.- Naini Tal; Pauri dist.- Satpuli; Uttarkashi dist.- Jalan, Dharasu.

\section{Aporrectodea caliginosa caliginosa (Savigny, 1826)}

Distribution: H.P.: Chamba dist.- Ehlla; Kinnaur dist.- Pooh; Kullu dist.- Manali, Kothi, Tirthan Valley (Shai Ropa, Kharongcha), Sainj Valley (Ropa); Lahaul \& Spiti dist.Madgram, Sissu; Shimla dist.- Chopal, Baldein, Kufri, Mashobra, Shimla, Jatog, Jakhu Hill, Narkanda, Bhogi Nalla Jubbal, Taradevi; Solan dist.- Chail, Janerghat, Blossom, Alampur, Nagali.

\section{Aporrectodea caliginosa trapezoides (Dugès, 1828)}

Distribution: J \& K: Anantnag dist.- Pahalgam, Anantnag; Budgam dist.- Ratsun; Doda dist.- Doda, Bhaderwah, Banihal; Srinagar dist.- Harwan, Srinagar. H.P.: Chamba dist.- Bhandal, Lower Bakrota, Daklotha, Hadsar, Ghararu, Rajour, Kilar, Chamba, Tissa, Rakh, Lakkar Mandi, Dalhousie, Khajjiar, 
Kailang, Kalatop, Khushnagari, Barmanh, Jukiyani, Bharmour, Malkauta; Kangra dist.- Jotla, Multhan; Kinnaur dist.- Karchham, Sholtu, Sangla; Kullu dist.- Vashistha Kund, Kasol, Kullu, Kothi, Marhi, Tirthan Valley (Kharongcha), Sainj Valley (Lapah); Lahaul \& Spiti dist.- Sissu, Tholang; Mandi dist.- Barot, Mandi; Shimla dist.- Kufri, Baldein, Jatog, Mashobra, Shilaroo, Khara Patthar, Shilghat, Shimla, Taradevi; Sirmour dist.- Sarahan, Habban, Haloni; Solan dist.Sadhupul, Jabli, Gambhar Bridge, Solan, Junga, Chail, Blossom. Uttaranchal: Almora dist.- Ranikhet, Soni, Punwa Nalla; Chamoli dist.- Joshimath, Mana, Joshimath-Tapovan Road, Garur Ganga, Helong; Champawat dist.- Champawat; Dehra Dun dist.- Chakarata, Mussoorie, Sahiya; Naini Tal dist.Naini Tal, Ramgarh; Pithoragarh dist.- Deval Thal; Tehri dist.Patli Devika; Uttarkashi dist.- Bhairo Ghati Lanka.

Remarks: Stephenson (1923) records this subspecies from Gilgit, Gurez, Gandarbal and Anchar Lake (Kashmir Valley). The identity of the specimens needs confirmation.

\section{Aporrectodea rosea rosea (Savigny, 1826)}

Distribution: J \& K: Baramulla dist.- Baba Rishi (Tangmarg); Budgam dist.- Chun; Srinagar dist.- Gandarbal. H.P.: Chamba dist.- Kharamukh, Sundla, Lakkar Mandi, Dalhousie, Khajjiar, Bhanjraroo, Kalatop; Kangra dist.- Mcleodganj; Kinnaur dist.- Pooh, Shipki La Road, Namgia; Kullu dist.Manali, Kullu, Kothi, Rahla Fall; Lahaul \& Spiti dist.- Kolang, Jahlman, Tholang, Goushal, Shansha, Mooring, Trilokinath, Sissu, Keylong; Mandi dist.- Barot, Baria; Shimla dist.- Chopal, Kufri, Mashobra, Shimla, Dhartoo Khari, Mashnu, Sanaba Forest Nursery, Ghanahatti, Kotkhai; Sirmour dist.- Habban, Kufar; Haripurdhar Fort; Solan dist.- Jabli, Nagali, Jhajha, Dagshai, Dharampur, Kasauli, Solan, Sadhupul, Chail. Uttaranchal: Chamoli dist.- Valley of Flowers, Joshimath; Dehra Dun dist.- Mussoorie; Naini Tal dist.- Naini Tal, Ramgarh; Pauri dist.- Lansdowne; Tehri dist.- Kaddukhal.

8. Dendrobaena hortensis (Michaelsen, 1890)

Distribution: H.P.: Shimla dist. - Shimla.

9. *Dendrobaena octaedra (Savigny, 1826)

Distribution: H.P.: Kullu dist.- Manali, Kothi, Jagatsukh, Solang Morrain; Mandi dist. - Barot.

\section{Dendrodrilus rubidus (Savigny, 1826)}

Distribution: J \& K: Baramulla dist.- Shit Garhi (Sonemarg). H.P.: Chamba dist.- Garola, Beauty, Lower Bakrota, Purthi Pangi, Daklotha, Lakkar Mandi, Hadsar, Dhansho, Kugti, Kailang, Ain Pokhar Nalla, Chhatri, Sara, Gatoth, Khajjiar, Kalatop, Rajour, Malkauta, Rota, Dinka; Kangra dist.- Multhan; Kinnaur dist.- Dabling Bridge, Karchham, Tapri, Pooh, Kalpa, Sholtu, Kamroo, Chango, Sangla, Rurtang, Namgia, Chaura, Rakchham-Chitkul, Nichar, Khursaran Nalla, Shialkhar, Rukti; Kullu dist.- Manali-Kothi Road, Manali, Rohtang-Marhi Road, Rahla Fall, Tirthan Valley (Kharongcha, Goshaini, Rolla, Grahani, Chhota Vaasu); Lahaul \& Spiti dist.- Koksar, Gondla, Lote, Sissu, Gopanthang, Thirot, Kirting, Kuth Bihar, Gramphu, Patsyoo, Kolang, Jahlman, Tholang, Bhayadi,
Trilokinath, Yangling, Keylong, Jispa, Shansha, Rappe, Sander Bridge, Udaipur; Mandi dist.- Barot, Ranuki Khud, Kao; Shimla dist.- Sarahan, Chopal, Kufri, Narkanda, Kinu, Pashad, Mashnu, Tutupani, Sanaba Forest Nursery, Kotkhai, Khara Patthar, Shilghat, Shimla, Junga, Matiana, Mashobra; Sirmour dist.- Nohra, Phagu, Tikattu, Dhartoo Khari. Uttaranchal: Almora dist.- Almora, Khati; Chamoli dist.Kedarnath, Tungnath, Vasu Dhara, Chopta, Hemkund, Valley of Flowers, Mana, Joshimath, Bedni; Dehra Dun dist.Chakrata, Musoorie; Naini Tal dist.- Gairal, Ramgarh, Naini Tal; Rudraprayag dist.- Phata, Trijuginaraian; Tehri dist.Dhanaulti; Uttarkashi dist.- Harsil, Bhairon Ghati Lanka, Uttarkashi.

\section{Eisenia fetida (Savigny, 1826)}

Distribution: H.P.: Chamba dist.- Lower Bakrota; Kangra dist.Dharamshala; Kinnaur dist.- Sholtu, Shong Tong, Nichar; Kullu dist.- Manali; Shimla dist.- Janog Ghat, Mashobra, Shimla, Narkanda, Kufri, Kartot, Ghanahatti, Shilghat; Sirmour dist.Rajgarh, Naina Tikkar, Nohra; Solan dist.- Solan, Arki, Oachghat, Chail, Kasauli. Uttaranchal: Chamoli dist.Govindghat, Joshimath, Padukeshwar; Dehra Dun dist.Mussoorie; Tehri dist.- Kaddukhal.

\section{Eiseniella tetraedra tetraedra (Savigny, 1826)}

Distribution: H.P.: Kinnaur dist.- Khursaran Nalla $(5 \mathrm{~km}$ from Kalpa); Shimla dist.- Janog Ghat, Thanedhar, Taradevi; Sirmour dist.- Giripul; Solan dist.- Ashwini Khud, Sadhupul, Oachghat, Chail Sanctuary. Uttaranchal: Naini Tal dist.- Naini Tal.

\section{Lumbricus castaneus (Savigny, 1826)}

Distribution: H.P.: Chamba dist.- Lower Bakrota; Shimla dist.Mashobra, Kufri.

14. Lumbricus terrestris Linnaeus, 1758 Distribution: H.P.: Shimla dist.- Shimla, Kufri.

15. Octolasion cyaneum (Savigny, 1826) Distribution: H.P.: Chamba dist.- Dalhousie, Kalatop; Kullu dist.- Manali, Kothi, Rahla Fall, Solang Morrain; Shimla dist.Kufri, Shimla, Mashobra, Taradevi. Uttaranchal: Dehra Dun dist.- Mussoorie.

16. Octolasion tyrtaeum (Savigny, 1826)

Distribution: J \& K: Anantnag dist.- Achabal; Budgam dist.Chadura; Srinagar dist.- Harwan. H.P.: Chamba dist.- Lower Bakrota, Lakkar Mandi, Dalhousie, Khajjiar, Matoonu, Kalatop, Rota; Kinnaur dist.- Dabling Bridge, Pooh, Kalpa, Sholtu, Chogaon, Sangla, Namgia, Ponda, Baro, Chaura, Sunnam, Rogi, Kaa, Shoulding, Shialkhar, Rukti, Rekong Peo, Shongtong, Nichar; Kullu dist.- Manali, Larji, Tirthan Valley (Shai Ropa, Kharongcha, Ropa, Barnag); Lahaul \& Spiti dist.Kirting, Lote, Tholang, Shansha; Mandi dist.- Barot; Shimla dist.- Chopal, Kufri, Baldein, Jatog, Shimla, Mashobra, Shilaroo, Narkanda, Dhartoo Khari, Rangori, Pashad, Mashnu, Kartot, Noon, Tutupani, Khaneti, Sanaba Forest 
Nursery, Ghanahatti, Gajta Nalla Kotkhai, Shilghat; Sirmour dist.- Dadahu; Solan dist.- Kasauli, Chail, Solan, Malon, Alampur, Jhajha, Blossom, Hinner, Nagali. Uttaranchal: Almora dist.- Jhoola Devi; Chamoli dist.- Joshimath, Padukeshwar; Dehra Dun dist.- Mussoorie, Bahmanpur, Sahastradhara, Chakrata; Naini Tal dist.- Bhim Tal, Ramgarh, Naini Tal; Tehri dist.- Dhanaulti, Kaddukhal, Patli Devika.

\section{Family: Almidae}

17. Glyphidrilus sp. (? gangeticus Gates, 1958)

Distribution: Uttaranchal: Naini Tal dist.- Naini Tal.

\section{Family: Ocnerodrilidae}

18. Malabaria levis (Chen, 1938)

Distribution: H.P.: Sirmour dist.- Bata Bridge (nr. Paonta Sahib).

\section{Ocnerodrilus occidentalis Eisen, 1878}

Distribution: J \& K: Doda dist.- Doda. H.P.: Bilaspur dist.- Bheri Ghat; Chamba dist.- Sundla, Rakh; Kangra dist.- Kangra; Kullu dist.- Manikaran; Mandi dist.- Mohan Ghatti; Solan dist.Solan, Jarash, Nalagarh; Una dist.- Lathiani, Nagain, Kuwadi. Uttaranchal: Dehra Dun dist.- Dehra Dun.

20. *Thatonia exilis Gates, 1945

Distribution: H.P.: Sirmour dist.- Bata Bridge (nr. Paonta Sahib).

21. *Thatonia gracilis Gates, 1942

Distribution: H.P.: Kangra dist.- Nurpur.

\section{Family: Acanthodrilidae}

22. Microscolex phosphoreus (Dugès, 1837)

Distribution: H.P.: Shimla dist.- Kukunala (3km W. of Kotkhai); Solan dist.- Ded Gharat, Kailar.

23. Plutellus sadhupulensis Julka and Paliwal, 1994

Distribution: H.P.: Solan dist.- Sadhupul.

\section{Family: Octochaetidae}

24. Dichogaster bolaui (Michaelsen, 1891)

Distribution: H.P.: Hamirpur dist.- Bhalet, Galod, Mair; Kangra dist.- Kangra, Jwalamukhi, Nurpur; Sirmour dist.- Rajgarh, Renuka Sanctuary; Solan dist.- Deli; Una dist.- Kotla Kalan, Lathiani, Nanavi. Uttaranchal: Dehra Dun dist.- Dehra Dun; Tehri dist.- Vyas Ji.

25. Eutyphoeus annandalei Michaelsen, 1907

Distribution: Uttaranchal: Naini Tal dist.- Bhim Tal.

26. Eutyphoeus incommodus (Beddard, 1901)

Distribution: J \& K: Kathua dist.- Changram. H.P.: Bilaspur dist.Kandhar, Maijpur, Goalthui, Bherighat; Chamba dist.- Rajpura, Chamba; Kangra dist.- Sadwan, Kangra, Jaritika, Dehra, Nurpur, Jasoor, Gangwal; Mandi dist.- Harabagh, Ner Chowk, Sunder Nagar, Mandi; Sirmour dist.- Paonta Sahib; Solan dist.- Kasauli; Una dist.- Kotla Kalan, Bharwain, Daulatpur, Ambota, Lathiani, Thana, Buhana, Nagain. Uttaranchal: Bageshwar dist.- Baijnath, Dhakuri; Chamoli dist.- Gairoli Patali, Bedni; Dehra Dun dist.- Dehra Dun; Pauri dist.- Dhikala-Sarapduli

\section{Road, Pauri, Chilla Forest.}

27. Eutyphoeus nainianus Michaelsen, 1907

Distribution: Uttaranchal: Chamoli dist.- Talwari; Naini Tal dist.Naini Tal; Pithoragarh dist.- Pabta.

28. Eutyphoeus nicholsoni (Beddard, 1901)

Distribution: H.P.: Sirmour dist.- Paonta Sahib. Uttaranchal:

Dehra Dun dist.- Doon Valley; Pauri dist.- Chilla Forest.

29. Eutyphoeus orientalis (Beddard, 1883)

Distribution: Uttaranchal: Dehra Dun dist.- Dehra Dun.

30. Eutyphoeus pharpingianus Michaelsen, 1907

Distribution: Uttaranchal: Pithoragarh dist.- Bastia.

31. Eutyphoeus waltoni Michaelsen, 1907

Distribution: J \& K: Jammu dist.- Parel (nr. Samba), Udhampur dist.- Mansar. H.P.: Bilaspur dist.- Swarghat, Bilaspur; Chamba dist.- Rajpura; Hamirpur dist.- Bhota, Deot Sidh; Kangra dist.Sadwan, Khajjan Khud, Darang, Bagli, Sehra, Nurpur, Gangwal, Dehra, Jasoor; Mandi dist.- Mandi, Ner Chowk, Sunder Nagar; Sirmour dist.- Gisani, Paonta Sahib; Una dist.Una, Bharwain, Kotla Kalan, Daulatpur, Kodi, Ambota, Lathiani, Thana, Nanavi, Buhana, Nagain. Uttaranchal: Almora dist.- Ranikhet; Dehra Dun dist.- Kandholi Forest, Dehra Dun; Pauri dist.- Ghasia Mahadev, Guthain, Dhikala-Sarapduli Road, Pauri, Chamdhar, Budhana, Dhikala Machan.

32. Lennogaster chittagongensis (Stephenson, 1917) Distribution: J \& K: Udhampur dist.- Mansar Lake. H.P.: Bilaspur dist.- Dukali; Chamba dist.- Sundla, Rajpura, Rakh, Chamba; Hamirpur dist.- Bhota; Kangra dist.- Sadwan, Khajjan Khud, Darang, Chobu, Kangra, Baijnath, Nurpur, Jasoor; Mandi dist.Badanu, Joginder Nagar, Mohan Ghatti, Ner Chowk, Sunder Nagar, Mandi, Hara Bagh, Dak Bagda, Tatta Pani; Sirmour dist.Sarahan, Gisani, Kulchet, Renuka, Jalal Khud (nr. Dadahu); Solan dist.- Malon, Sadhupul, Oachghat, Dharampur, Gaura, Kunihar; Una dist.- Daulatpur, Ambota, Lathiani, Thana, Nagain.

33. (?) Lennogaster parvus (Fedarb, 1898) Distribution: Uttaranchal: Dehra Dun.

34. Lennogaster pusillus (Stephenson, 1920) Distribution: H.P.: Chamba dist.- Rakh; Kangra dist.- Nurpur; Mandi dist.- Dak Bagda, Badanu; Shimla dist.- Kotkhai; Solan dist.- Solan. Uttaranchal: Almora dist.- Chaukni; Pauri dist.Dhikala.

35. Lennogaster yeicus (Stephenson, 1931)

Distribution: H.P.: Kangra dist.- Mohan Ghatti; Mandi dist.Dak Bagda, Kara Forest (nr. Hanogi); Shimla dist.- Kotkhai. 36. Octochaetona beatrix (Beddard, 1902)

Distribution: J \& K: Jammu dist.- Budhwani. H.P.: Bilaspur dist.- Kandhor, Bherighat; Chamba dist.- Rajpura, Chamba, Rakh; Hamirpur dist.- Bhota; Kangra dist.- Nurpur, Jasoor, Gangwal, Sadwan, Khajjan Khud, Kedara, Dehra; Mandi dist.- 
Joginder Nagar, Dak Bagda, Sunder Nagar, Harabagh, Tatta Pani; Sirmour dist.- Sarahan, Tikkar, Gisani, Paonta Sahib, Renuka Sanctuary; Solan dist.- Kunihar, Subathu, Kasauli, Sadhupul, Oachghat; Una dist.- Bharwain, Daulatpur, Ambota, Nanavi. Uttaranchal: Dehra Dun dist.- Rishikesh, Kandholi Forest, Dehra Dun; Naini Tal dist.- Gairal; Pauri dist.- Jwalpa Devi.

37. Ramiella bishambari (Stephenson, 1914)

Distribution: H.P.: Kangra dist.- Nurpur, Jasoor; Shimla dist.Pulbahal; Solan dist.- Kunihar. Uttaranchal: Naini Tal dist.Haldwani.

\section{Family: Megascolecidae}

38. Amynthas alexandri (Beddard, 1900)

Distribution: H.P.: Hamirpur dist.- Sujanpur Tihra; Kangra dist.Kedara, Palampur; Mandi dist.- Joginder Nagar, Sunder Nagar; Sirmour dist.- Dadahu; Una dist.- Nanavi. Uttaranchal: Dehra Dun dist.- Gujrara, Sahastradhara, Nehrugram; Naini Tal dist.- Rani Bagh; Pauri dist.- Khankra.

\section{Amynthas corticis (Kinberg, 1867)}

Distribution: J \& K: Budgam dist.- Ratsun, Beerwah; Srinagar dist.- Harwan, Srinagar. H.P.: Bilaspur dist.- Dukali; Chamba dist.- Bhandal, Pukhari, Sundla, Rajpura, Rakh, Dalhousie, Khajjiar, Khushnagari, Gajnui, Chaned, Jukiyani, Saho; Hamirpur dist.- Bhalet, Mundkhar; Nadaun; Kangra dist.Dharamshala, Kotla, Kangra, Palampur, Neugal Khud, Churan Khud, Multhan, Tang-Baroi, Bid, Binwa Nagar, Sidhpur, Bhagsu Nag, Gaggal; Kinnaur dist.- Tapri; Kullu dist.- Kullu, Ramshed, Vashisth Kund, Kasol, Bhuntar, Badanu, Larji, Tirthan Valley (Shai Ropa, Goshaini, Ropa), Sainj Valley (Ropa); Lahaul \& Spiti dist.- Keylong; Mandi dist.- Joginder Nagar, Dak Bagda, Hara Bagh, Tattapani, Barot; Shimla dist.- Shimla, Baghara, Mashobra, Pashad, Kartot, Suni, Chopal, Silaroo, Chhaila; Sirmour dist.- Rajgarh, Habban, Naina Tikkar, Dadahu, Chandni, Laaldang, Sarahan, Renuka Sanctuary, Giripul, Ranhat; Solan dist.- Koti, Dharampur, Chail, Sadhupul, Kuthar, Parwanu, Gambhar Bridge, Arki, Kasauli, Solan, Malon, Subathu, Deothal, Lugoan Bridge, Bajdol, Shilli Forest, Barog, Sadhupul, Nagali. Uttaranchal: Almora dist.Kausani, Chaukni, Sheraghat, Lakhidiyar, Soni; Bageshwar dist.- Bageshwar, Garur, Jogeshwar, Baijnath; Chamoli dist.Bhimtoli, Joshimath, Mandal, Pandukeshwar, Mana, Govindghat; Champawat dist.- Lohaghat, Bed Bagad; Dehra Dun dist.- Amlawa River bed, Kalsi, Rajpur, Ramgash; Naini Tal dist.- Bhim Tal, Naini Tal, Sat Tal, Ramgarh, Mohan, Malani; Pauri dist.- Lansdowne, Kanda, Pauri, Malan Sanctuary, Chamdhar, Gumkhal; Pithoragarh dist.- Didihat, Thal, Jharia Gad, Pithoragarh, Berinag, Dhamrikhet, Arca; Rudra Prayag dist.- Phata, Augastmani, Narainkoti, Trijuginarain, Tehri dist.- Kirtinagar, Kaddukhal, Pokhal; Uttarkashi dist.Uttarkashi, Maneri, Raturi Sera, Jashyra, Badyar Gad, Mori, Dunda.

40. Amynthas gracilis (Kinberg, 1867)

Distribution: H.P.: Kangra dist.- Palampur. Uttaranchal: Chamoli dist.- Ramnee; Dehra Dun dist.- Dehra Dun.

\section{Amynthas morrisi (Beddard, 1892)}

Distribution: J \& K: Doda dist.- Bhadarwah; Poonch and Srinagar districts. H.P.: Bilaspur dist.- Nauni; Chamba dist.- Hardaspur, Rajpura; Kangra dist.- Palampur; Shimla dist.- Suni; Sirmour dist.- Nahan; Solan dist.- Dharampur, Solan, Sadhupul, Subathu, Deothal. Uttaranchal: Dehra Dun dist.- Raipur, Dehra Dun.

42. Metaphire anomala (Michaelsen, 1907)

Distribution: H.P.: Kangra dist.- Dadh, Kangra, Palampur; Mandi dist.- Joginder Nagar; Sirmour dist.- Dadahu. Uttaranchal: Chamoli dist.- Nandprayag, Thirpali; Dehra Dun dist.- Sahastradhara, Dehra Dun, Lachhiwala, Ghattikhola, Gujrara, Mussoorie Forest Range, Barkot; Naini Tal dist.Naukuchia Tal; Pauri dist.- Dhikala-Kanda Road, Tehri dist.Deoprayag.

\section{Metaphire birmanica (Rosa, 1888)}

Distribution: H.P.: Hamirpur dist.- Tikkar, Sujanpur Tihra; Kangra dist.- Darang, Kangra; Mandi dist.- Joginder Nagar, Baijnath, Dak Bagda, Ner Chowk, Sunder Nagar; Sirmour dist.- Renuka Sanctuary; Solan dist.- Solan. Uttaranchal: Bageshwar dist.- Garur, Kapkote; Chamoli dist.- Pipalkoti, Kuher, Nandprayag; Dehra Dun dist.- Timli, Dehra Dun, Mussoorie, Nehrugram, Ghattikhola, Kandholi Forest; Naini Tal dist.- Naini Tal, Deochauri, Naukuchia Tal; Pauri dist.Dhikala-Kanda Road, Dhikala-Sarapduli Road; Rudraprayag dist.- Rudraprayag.

\section{Metaphire houlleti (Perrier, 1872)}

Distribution: J \& K: Udhampur dist.- Katra, Udhampur; H.P.: Bilaspur dist.- Dukali; Hamirpur dist.- Sujanpur Tihra, Jungle Beri, Bhalet; Kangra dist.- Dehra, Nurpur, Jasoor, Sadwan, Baijnath, Darang, Chobu, Kedara, Ganoh, Palampur, Chimbalhar; Mandi dist.- Joginder Nagar, Mohan Ghatti, Gumma, Dak Bagda, Ner Chowk, Sunder Nagar, Hara Bagh, Tattapani, Jeori, Mandi; Shimla dist.- Suni; Sirmour dist.Banethi, Renuka Sanctuary, Dadahu, Ajoli, Daluwala, Shirumella; Solan dist.- Gaura, Gambhar Bridge, Oachghat, Dharampur, Kasauli, Chhawasa, Solan; Una dist.- Ambota, Thana, Nanavi. Uttaranchal: Almora dist.- Soni, Chitai; Chamoli dist.- Thirpali; Champawat dist.- Champawat; Dehra Dun dist.- Dehra Dun, Sahiya, Nehrugram, Ghattikhola, Lalpul, Timli Forest, Sahastradhara, Rishikesh, Phanduwala, Gujrara; Naini Tal dist.- Gagasat Sultan, Ranibagh, Bhowali, Dhangarhi, Naukuchia Tal, Sat Tal, Kala Dungi, Bhim Tal; Pithoragarh dist.- Thal, Jharia Gad, Oriti, Ravti Nalla, Kolika; Rudraprayag dist.- Rudraprayag; Tehri dist.- Kirtinagar, Aglar River, Gulabrai, Chamba.

\section{Metaphire posthuma (Vaillant, 1868)}

Distribution: J \& K: Jammu dist.- Barpur; Udhampur dist.Udhampur. H.P.: Chamba dist.- Sundla, Rajpura; Kangra dist.Jasoor; Mandi dist.- Tattapani, Jeori; Sirmour dist.- Dadahu, Renuka Sanctuary; Una dist.- Kodi. Uttaranchal: Dehra Dun dist.- Kansro Forest, Easthope Town, Dehra Dun, Satyanarayan, Lachhiwala; Tehri dist.- Deoprayag. 
46. Perionyx bainii Stephenson, 1915

Distribution: H.P.: Chamba dist.- Bhandal, Khajjiar, Banikhet; Kangra dist.- Multhan, Dharamshala; Kinnaur dist.- Tapri, Sholtu, Chogaon, Nichar, Baro, Chaura, Khursaran Nalla; Kullu dist.- Juanu Khud, Manali, Rahla Fall, Kothi, Tirthan Valley (Ropa, Barnagi, Kharongcha, Rolla, Poocha, Grahani, Shilt, Chhota Vaasu), Sainj Valley (Ropa, Neoli, Marm, Lapah, Denga Pul, Sakti, Dolnu, Shanghar), Jiwanal; Mandi dist.Barot; Shimla dist.- Sarahan, Pulbahal, Kotgarh, Jarol, KotiBadhog, Kunni, Gaura, Kholidhar, Tutupani, Thanedhar, Khara Patthar, Shimla; Sirmour dist.- Nohra, Renuka Sanctuary, Phagu, Habban, Rajgarh, Haripurdhar, Jarva, Nohradhar, Rondi Nalla; Solan dist.- Junga, Chail Sanctuary, Hinner. Uttaranchal: Almora dist.- Kausani, Chaukni, Almora; Chamoli dist.- Joshimath, Mandal, Dugal Batta, Tungnath, Padukeshwar; Dehra Dun dist.- Chakrata; Pithoragarh dist.Ghatkola Nalla; Rudraprayag dist.- Guptakashi; Uttarkashi dist.- Kutnaur, Rawain Forest Range, Syana Chatti, Barnigad Kuwa.

47. Perionyx barotensis Julka and Paliwal, 1993

Distribution: H.P.: Kullu dist.- Tirthan Valley (Barnagi, Ropa, Rolla), Sainj Valley (Ropa); Mandi dist.- Barot.

\section{Perionyx excavatus Perrier, 1872}

Distribution: H.P.: Bilaspur dist.- Nihari Forest Plantation, Bamta, Kandror, Kanaula; Chamba dist.- Lower Bakrota, Sundla, Rakh; Hamirpur dist.- Hera Nagar, Sujanpur Tihra, Galod, Bharnani; Kangra dist.- Nurpur, Bhagsu Nag, Banoi, Palampur, Chimbalhar; Mandi dist.- Pandoh, Gumma, Barot; Shimla dist.- Taradevi, Baghara, Jatog, Shimla, Rajpur; Sirmour dist.- Nohra, Naina Tikkar, Dhamla, Dadahu, Kialtu, Renuka Sanctuary, Chandni, Rajgarh, Sataun, Paonta Sahib, Shillai; Solan dist.- Sadhupul, Patta, Solan, Parwanu, Chhawasa, Jarash, Lugoan Bridge, Ramshahar, Chail, Hinner; Una dist.Thana, Nanavi. Uttaranchal: Almora dist.- Almora, Sheraghat; Chamoli dist.- Joshimath; Champawat dist.- Phorti, Sandev; Dehra Dun dist.- Phanduwala, Dehra Dun, Satyanarain, Kansro Forest, Motichur Rao; Naini Tal dist.- Bhowali, Naini Tal, Mohan; Pauri dist.- Khankara, Chamdhar, Kanda Nala; Uttarkashi dist.- Barnigad Kuwa.

\section{Perionyx nainianus (Michaelsen, 1907)}

Distribution: Uttaranchal: Almora dist.- Dhakuri, Khati; Chamoli dist.- Wan, Gairoli Patali, Bedni; Naini Tal dist.- Naini Tal; Pithoragarh dist- Ghattabagur, Kulagarh; Rudraprayag dist.Augastmani, Guptakashi.

50. Perionyx sansibaricus Michaelsen, 1891

Distribution: H.P.: Kangra dist.- Holta (nr. Palampur); Solan dist.- Parwanu. Uttaranchal: Dehra Dun dist.- Clemant Town, Easthope Town.

51. Perionyx simlaensis (Michaelsen, 1907)

Distribution: H.P.: Sirmour dist.- Renuka Sanctuary, Dadahu,

Paonta Sahib, Bata Bridge; Solan dist.- Dharampur, Ramshahar. Uttaranchal: Dehra Dun dist.- Ramgash.

\section{ReFERENCES}

Bourne, A.G. (1889). On certain earthworms from the western Himalayas and Dehra Dun. Journal of the Asiatiic Society of Bengal 58: 110-117.

Cernosvitov, L. (1937). On a collection of Indian earthworms of the family Lumbricidae. Records of the Indian Museum 39: 105-111.

Fedarb, S.M. (1898). On some earthworms from British India. Proceedings of the Zoological Society of London 1898: 445-450.

Gates, G.E. (1945). Another species of Pheretima from India. Science and Culture, Calcutta 10(9): 403.

Gates, G.E. (1951). On the earthworms of Saharanpur, Dehra Dun and some Himalayan hill stations. Proceedings of the National Academy of Sciences of India (B) 21: 16-22.

Halder, K.R. and G.C. Gosh (1997). Annelida, pp.31-34. In: Fauna of Nanda Devi Biosphere Reserve: Fauna of Conservation Areas 9. Zoological Survey of India, Calcutta.

Julka, J.M. (1979). First record of Lumbricus castaneus (Savigny) from India (Lumbricidae: Oligochaeta). Journal of the Bombay Natural History Society 76(1): 191-192.

Julka, J.M. (1981). Anthropochorous earthworms of Lahaul Valley (Himachal Pradesh) with notes on their ecology. In: Veeresh, G.K. (ed.) Progress in Soil Biology and Ecology in India, UAS Technical Series 37: 69-76. University of Agricultural Sciences, Bangalore.

Julka, J.M. (1988). The Fauna of India and the adjacent countries. Megadrile Oligochaeta (Earthworms). Haplotaxida: Lumbricina: Megascolecoidea: Octochaetidae. Zoological Survey of India, Calcutta, 400pp+xiv.

Julka, J.M. (1995). Oligochaeta, pp.17-22. In: Himalayan Ecosystem Series: Fauna of Western Himalaya, Part 1: Uttar Pradesh. Zoological Survey of India, Calcutta.

Julka, J.M.(1999-Unpublished). Biodiversity assessment of Annelida and Mollusca in the Great Himalayan National Park. FREE-GHNP Research Project, Wildlife Institute of India, Dehra Dun.

Julka, J.M. and R. Paliwal (1993). A new species of Perionyx Perrier (Megascolecidae, Oligochaeta) from northwest Himalaya, India. Journal of the Bombay Natural History Society 90(3): 461-462.

Julka, J.M. and R. Paliwal (1994). On a new species of Plutellus Perrier (Acanthodrilidae: Oligochaeta) from northwest Himalaya, India. Research Bulletin of the Panjab University (Science) 44(I-IV): 217-220.

Julka, J.M. and R. Paliwal (1995). First records of Microscolex phosphoreus and Malabaria levis (Oligochaeta: Acanthodrilidae and Ocnerodrilidae) from India. Megadrilogica 6(6): 60-62.

Julka, J.M. and R. Paliwal (2000). Oligochaeta, pp.21-25. In: Fauna of Renuka Wetland: Wetland ecosystem series 2. Zoological Survey of India, Calcutta. Michaelsen, W. (1907). Neue Oligochäten von Vorder-Indien, Ceylon, birma, und den Andaman-Inseln. Jahrbuch der Hamburgischen Wissenschaftlichen Anstalten 24: 143-188.

Michaelsen, W. (1909). The Oligochaeta of India, Nepal, Ceylon, Burma and the Andaman Islands. Memoirs of the Indian Museum 1: 103-253.

Sharma, B.D. and T.K. Kaul (1974). Note on the distribution of four genera of earthworms in J \& K State. Indian Journal of Animal Research 8(1): 46.

Soota, T.D. (1970). A taxo-ecological study of the earthworm fauna of Doon Valley. Records of the Zoological Survey of India 64: 173-184.

Soota, T.D. and K.R. Halder (1980). On some earthworms from western Himalayas. Records of the Zoological Survey of India 76: 195-205.

Stephenson, J. (1914). On a collection of Oligochaeta, mainly from Northern India. Records of the Indian Museum 10: 321-365.

Stephenson, J. (1916). On a collection of Oligochaeta belonging to the Indian Museum. Records of the Indian Museum 12: 299-354.

Stephenson, J. (1922). Some earthworms from Kashmir, Bombay, and other parts of India. Records of the Indian Museum 24: 427-443.

Stephenson, J. (1923). Oligochaeta. The Fauna of British India, including Ceylon and Burma. Taylor and Francis, London, 518pp+xxiv.

\section{ACKNOWLedgement}

The authors are grateful to the Director, Zoological Survey of India, Kolkata and Officer-in-charge, High Altitude Zoology Field Station, Solan for providing necessary facilities to undertake this work. 